\title{
Evaluation of Three Different Bleaching Agents in Permanent and Primary Teeth: An In Vitro Study
}

\author{
Manisha Behl ${ }^{1}$, Arun K Patnana ${ }^{2}$, Vikram Khanna ${ }^{3}$, Kirti Chaudhry ${ }^{4}$
}

\begin{abstract}
Aim: To evaluate the effectiveness of three different bleaching agents in permanent and primary teeth.

Materials and methods: The study was done on 60 freshly extracted single-rooted teeth (30 permanent and 30 primary incisors) with intact coronal portion. The teeth were artificially stained with whole blood as the staining medium in a high-speed centrifuge. The discolorations were determined by the use of 3D Master Vita shade guide. The conventional endodontic treatment procedures were completed in all the test samples. The test samples were divided into group I (permanent teeth) and group II (primary teeth). The test samples were further divided into three subgroups according to the experimental materials used for bleaching [group IA-10\% carbamide peroxide (CP), group IIB-9.5\% hydrogen peroxide (HP), and group IIIC-10\% sodium perborate (SP)]. The samples were evaluated at 7 days and 14 days after the completion of intracoronal bleaching procedure.

Results: The CP group showed lowest shade values, and SP group showed the highest shade values after 14 days in both permanent and primary teeth. The CP showed the significant difference in the shade at 7 days and 14 days in permanent teeth. No statistically significant difference was observed between the three experimental at the 7 days and 14 days in the primary teeth.

Conclusion: The CP is effective in permanent teeth after 7 days and 14 days of intracoronal bleaching. The three experimental materials are equally effective in the primary teeth bleaching after 7 days and 14 days.
\end{abstract}

Keywords: Carbamide peroxide, Deciduous teeth, Experimental study, Permanent teeth, Tooth bleaching.

International Journal of Clinical Pediatric Dentistry (2020): 10.5005/jp-journals-10005-1721

\section{INTRODUCTION}

The dental professionals were perplexed for the treatment of discolored teeth since last two hundred years and have tried different chemical methods to remove various types of discolorations in the teeth. ${ }^{1}$ The tooth discolorations are classified as extrinsic and intrinsic, where the extrinsic stains can be removed with the routine prophylactic measures in the dental office. $^{2}$ The intrinsic discolorations in the tooth might result from penetration of discoloring agents in the dentinal tubules, systemic medications, excess water fluoridation, or due to by-products of the body such as bilirubin released into the dentinal tubules during illness. ${ }^{3}$ The intrinsic stains can be treated by various measures such as internal bleaching of nonvital teeth, external bleaching of vital teeth, microabrasion of enamel and prosthetic rehabilitation by crowns and veneers. ${ }^{4}$ The intracoronal bleaching is an established, simple, cost-effective, and conservative method for improving the color of discoloured teeth in permanent and primary teeth. The advantage like minimal patient compliance makes the intracoronal bleaching technique more applicable in the children and young adolescents. ${ }^{1}$

The hydrogen peroxide (HP) (30\% and 35\%), sodium perborate (SP), carbamide peroxide (CP) $(10 \%, 20 \%, 35 \%)$ are the most commonly used bleaching agents for permanent and primary teeth. ${ }^{5}$ Hydrogen peroxide is a strong oxidizing agent that can penetrate dentin and releases oxygen, which further breaks the double bonds of organic and inorganic compounds inside the dentinal tubules causing decolorization of the stains. ${ }^{6}$ One of the main undesirable consequences of intracoronal bleaching with HP was external cervical root resorption of the tooth. The excessive diffusion of HP into the periradicular tissues, possibly through cemental defects, was the possible reason behind the cervical root
${ }^{1,3}$ Department of Oral Medicine and Radiology, King George's Medical University, Lucknow, Uttar Pradesh, India

${ }^{2,4}$ Department of Dentistry, All India Institute of Medical Sciences, Jodhpur, Rajasthan, India

Corresponding Author: Kirti Chaudhry, Department of Dentistry, All India Institute of Medical Sciences, Jodhpur, Rajasthan, India, Phone: +91 8003993889, e-mail: chaudhry_kirti@yahoo.com

How to cite this article: Behl M, Patnana AK, Khanna V. Evaluation of Three Different Bleaching Agents in Permanent and Primary Teeth: An In Vitro Study. Int J Clin Pediatr Dent 2020;13(2):130-135.

Source of support: Nil

Conflict of interest: None

resorptions in the bleached teeth. Although HP was a potent and efficient bleaching agent, there was still a controversy regarding its safety and possible adverse effects on the teeth. ${ }^{7}$

Sodium perborate is another commonly used intracoronal bleaching agents, which can be used alone or in combination with HP. Though the SP produces no adverse effects, it may take longer time to produce appreciable color change in the affected tooth. ${ }^{5}$ The different concentrations of the $\mathrm{CP}$ have been extensively used in the bleaching of the discolored teeth. ${ }^{8}$ The various experimental and clinical studies have shown that the $\mathrm{CP}$ shows significant color change by the intracoronal applications in discolored teeth. ${ }^{9}$ The $\mathrm{CP}$ carries low risk of external root resorptions than the HP and SP after the completion of bleaching in discolored teeth. ${ }^{10}$

Pediatric literature had indicated that $72 \%$ of primary teeth after concussion injuries remained asymptomatic with no clinical or radiographic abnormalities. ${ }^{11}$ However, there was still some debate exist about discolorations and pulpal status in primary teeth. ${ }^{12}$ 
Hence, bleaching was considered as a reasonable treatment option for discolored primary teeth with no clinical and radiographic abnormalities. Though different in vitro studies have compared various bleaching agents in permanent and primary teeth separately, no studies have compared the effectiveness of different bleaching agents between permanent and primary teeth. Hence, the primary objective of the present study was to evaluate the effective bleaching agent in primary and permanent teeth and the secondary objective was to evaluate the differences in the effectiveness of each bleaching agent in permanent and primary teeth.

\section{Materials and Methods}

The present experimental study was conducted in the Department of Pediatric Dentistry in a dental college and hospital. The present study includes a total of 60 freshly extracted teeth (group I = 30 permanent and group $\|=30$ primary teeth) with intact crowns. The extracted teeth were collected from the Department of Oral and Maxillofacial Surgery of the same dental college and hospital. The soft tissues and debris covering the root surfaces was cleaned with hand scalers. The teeth were immersed in $5 \%$ sodium hypochlorite for 1 hour and then stored in physiological saline until use.

The stored samples were stained using whole blood as the staining medium in a high-speed centrifuge. Each tooth was stored in a test tube filled one-third of the way with whole blood and incubated at $37^{\circ} \mathrm{C}$ for 4 hours. Each tooth was then centrifuged at 2,500 rpm for 20 minutes, twice daily for 3 consecutive days to enhance the penetration of hemolyzed red blood cells into the dentinal tubules. The precipitate, which got collected at the bottom after the centrifugation procedure, was removed, and the teeth immersed were in remaining hemoglobin-rich hemosylate left in the upper part of the test tube for a further 3 days, centrifuging it twice daily as previously described. The resultant discolored teeth obtained were then washed with distilled water.
After staining the extracted teeth with centrifugation method the stained teeth were assessed for their color change as compared to the prestained state by means of Vita 3D Master Shade guide. The color change was recorded, and the stained teeth were photographed. All the stained teeth were assigned with values, which came out to be 4 and 5 in terms of their value ratings as coded by the Vita 3D shade guide.

After standard access cavity preparation, the root canals were cleaned and shaped using 5\% sodium hypochlorite and normal saline for irrigation. Biomechanical preparation was done with $\mathrm{K}$ files, and the canals were subsequently enlarged using step-back technique in permanent teeth and selective filing technique in deciduous teeth. This was followed by obturation with zinc oxide eugenol in primary teeth and gutta-percha with root canal sealer using lateral condensation in permanent teeth.

The teeth were randomly subdivided into three experimental groups, according to the bleaching material used in the pulp chamber in both permanent and primary teeth (subgroup $A$ : $10 \%$ CP gel; subgroup B: $9.5 \%$ HP gel; subgroup C: $10 \%$ SP paste) (Fig. 1). Two millimeters of root canal filling was removed below the vestibular cementoenamel junction, and the remaining obturating material was covered with a 1-mm-thick cervical seal of cavit (Fig. 2). Around $0.04 \mathrm{~mL}$ of bleaching agent was syringed into the access cavity of the tooth and then sealed with temporary sealing material. After 7 days, color of bleached teeth was determined. The original bleaching agent was then washed away, and a fresh portion of bleaching agent was syringed into the access cavity. The teeth were then again evaluated for their shade change after another 7 days. All the data were collected and tabulated. All these values were further statistically analyzed for evaluation.

\section{Results}

Table 1 shows the mean values obtained for shade lightening of all the permanent and primary teeth on 0,7 , and 14 days.
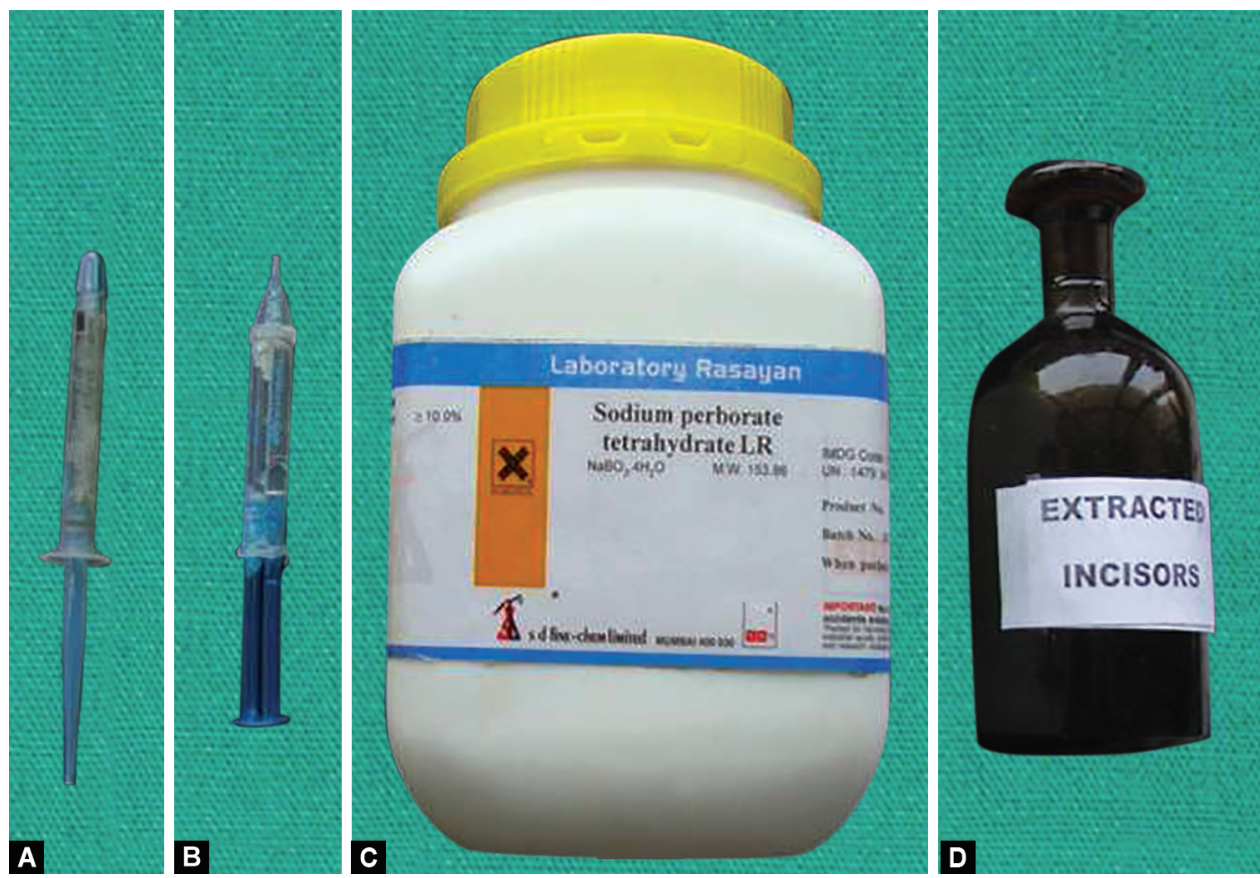

Figs $1 A$ to D: Experimental materials used in the present study: (A) 10\% carbamide peroxide; (B) 9.5\% hydrogen peroxide; (C) Sodium perborate; (D) Extracted incisors 

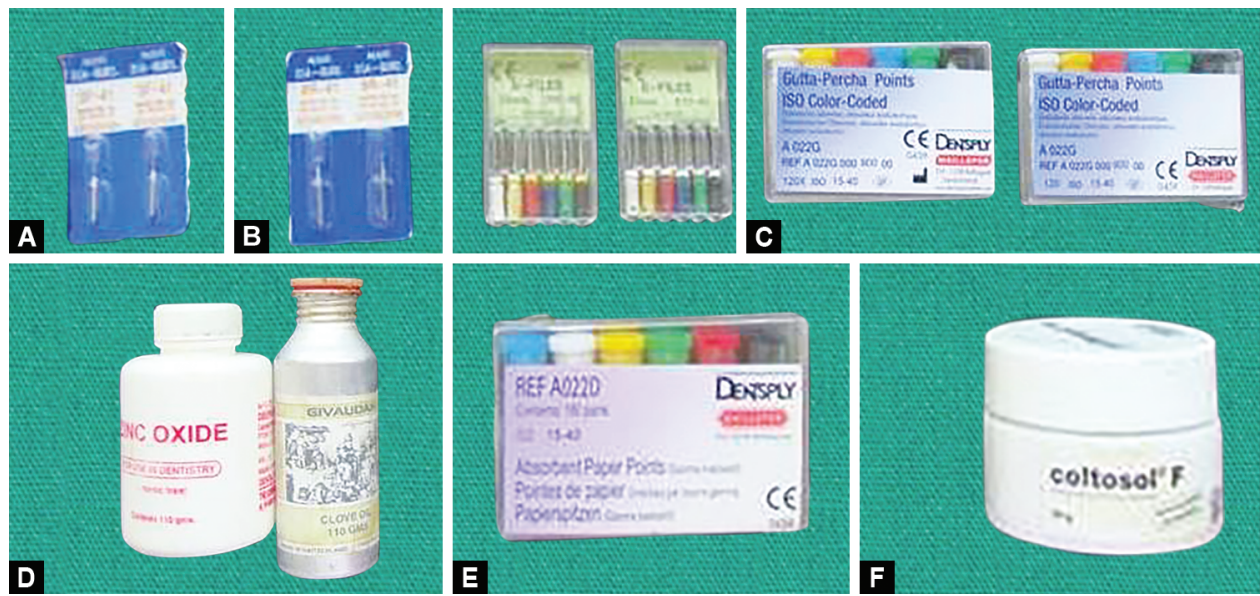

Figs 2A to G: Materials used for the conventional root canal treatment in the present study: (A) Round burs; (B) Straight fissure burs; (C) K files; (D) Gutta percha points; (E) Zinc oxide eugenol; (F) Absorbant paper points; (G) Temporary cement

Table 1: Mean values of the color change after 7 days and 14 days in the experimental groups

\begin{tabular}{llrrr}
\hline & & \multicolumn{3}{c}{ Mean values } \\
\cline { 3 - 4 } Group & Subgroups & \multicolumn{1}{c}{ Oday } & \multicolumn{1}{c}{7 days } & \multicolumn{1}{c}{14 days } \\
\hline Permanent teeth (group I) & CP (group IA) & $9.2 \pm 1.03$ & $3.9 \pm 1.28$ & $2.6 \pm 1.07$ \\
$n=30$ & HP (group IB) & $10.1 \pm 1.59$ & $6.9 \pm 1.73$ & $6.2 \pm 1.99$ \\
& SP (group IC) & $11 \pm 2.36$ & $9 \pm 2.58$ & $8.5 \pm 2.41$ \\
Primary teeth (group II) & CP (group IIA) & $10.7 \pm 2.11$ & $8.4 \pm 1.57$ & $7.2 \pm 1.61$ \\
$n=30$ & HP (group IIB) & $10.5 \pm 1.95$ & $8.6 \pm 2.06$ & $7.9 \pm 2.33$ \\
& SP (group IIC) & $11.5 \pm 2.17$ & $9.2 \pm 1.93$ & $8.3 \pm 1.7$ \\
\hline
\end{tabular}

Table 2: Shade variations in permanent and primary teeth with carbamide peroxide in at 0, 7, and 14 days

\begin{tabular}{llrccc}
\hline Days & Teeth & Mean $\pm S D$ & Mean square value & Fvalue & $p$ value \\
\hline 0 & Permanent teeth & $9.2 \pm 1.03$ & 11.25 & 4.07 & 0.06 \\
& Primary teeth & $10.7 \pm 2.11$ & & & \\
7 & Permanent teeth & $3.9 \pm 1.28$ & 101.25 & 48.86 & $0.00^{*}$ \\
& Primary teeth & $8.4 \pm 1.57$ & & & \\
\multirow{2}{*}{14} & Permanent teeth & $2.6 \pm 1.07$ & 105.8 & 56.01 & $0.00^{*}$ \\
& Primary teeth & $7.2 \pm 1.61$ & & & \\
\hline
\end{tabular}

${ }^{*} p$ value of less than 0.05 was considered as significant

Table 3: Effectiveness of hydrogen peroxide in permanent and primary teeth at 0,7 , and 14 days

\begin{tabular}{llrlll}
\hline Days & Teeth & Mean $\pm S D$ & Mean square value & Fvalue & $p$ value \\
\hline 0 & Permanent teeth & $10.1 \pm 1.59$ & 3.19 & 0.25 & 0.62 \\
& Primary teeth & $10.5 \pm 1.95$ & & & \\
7 & Permanent teeth & $6.9 \pm 1.72$ & 14.45 & 3.98 & 0.06 \\
& Primary teeth & $8.6 \pm 2.06$ & & & \\
\multirow{2}{*}{14} & Permanent teeth & $6.25 \pm 1.99$ & 14.45 & 3.08 & 0.09 \\
& Primary teeth & $7.9 \pm 2.33$ & & & \\
\hline
\end{tabular}

${ }^{*} p$ value of less than 0.05 was considered as significant

The CP group shows lowest shade values (group $I A=2.6$ and group $\| \mathrm{A}=7.2$ ) and SP group showed the highest shadevalues (group $\mathrm{IC}=8.5$ and group $\| \mathrm{C}=8.3$ ) after 14 days in both permanent and primary teeth.

Table 2 shows the shade variations in permanent and primary teeth with $\mathrm{CP}$ at 0,7 , and 14 days intervals. The shade for the both permanent and primary teeth shows no significant difference at the start of the study $(p=0.06)$. The permanent and primary teeth have showed the significant difference in the shade at 7 days $(p=0.00)$ and 14 days $(p=0.00)$ after the placement of medicaments.

Table 3 shows the shade variations in permanent and primary teeth with HP at 0,7 , and 14 days intervals. The shade for the both permanent and primary teeth shows no significant difference at the start of the study $(p=0.62)$. The permanent and primary teeth have showed no the significant difference even at the 7 days $(p=0.06)$ and 14 days $(p=0.09)$ after the placement of medicaments. 
Evaluation of Bleaching Agents in Teeth

Table 4: Effectiveness of sodium perborate in permanent and primary teeth at 0, 7, and 14 days

\begin{tabular}{llrlll}
\hline Days & Teeth & Mean $\pm S D$ & Mean square value & Fvalue & $p$ value \\
\hline 0 & Permanent teeth & $11 \pm 2.17$ & 1.25 & 0.24 & 0.63 \\
& Primary teeth & $11.5 \pm 2.17$ & & & \\
7 & Permanent teeth & $9 \pm 2.58$ & 0.20 & 0.04 & 0.85 \\
& Primary teeth & $9.2 \pm 1.93$ & & & \\
\multirow{2}{*}{14} & Permanent teeth & $8.5 \pm 2.41$ & 0.20 & 0.05 & 0.83 \\
& Primary teeth & $8.3 \pm 1.70$ & & & \\
\hline
\end{tabular}

Table 5: Shade variations by the three experimental materials at 0,7 and 14 days in permanent teeth

\begin{tabular}{|c|c|c|c|c|c|}
\hline Days & Sub group & Mean $\pm S D$ & Mean square value & Fvalue & $p$ value \\
\hline \multirow[t]{3}{*}{0} & $\mathrm{CP}$ & $9.2 \pm 1.03$ & 8.1 & 2.65 & 0.89 \\
\hline & $\mathrm{HP}$ & $10.1 \pm 1.59$ & & & \\
\hline & $\mathrm{SP}$ & $11 \pm 2.35$ & & & \\
\hline \multirow[t]{3}{*}{7} & $\mathrm{CP}$ & $3.9 \pm 1.28$ & 65.7 & 17.42 & 0.00 \\
\hline & HP & $6.9 \pm 1.72$ & & & \\
\hline & SP & $9 \pm 2.58$ & & & \\
\hline \multirow[t]{3}{*}{14} & $\mathrm{CP}$ & $2.6 \pm 1.07$ & 88.43 & 24.24 & 0.00 \\
\hline & HP & $6.2 \pm 1.99$ & & & \\
\hline & SP & $5.7 \pm 3.08$ & & & \\
\hline
\end{tabular}

Table 6: Color change variations by the three experimental materials at 0,7 , and 14 days in primary teeth

\begin{tabular}{llllll}
\hline Days & Subgroup & Mean \pm SD & Mean square value & Fvalue & pvalue \\
\hline 0 & CP & $10.7 \pm 2.11$ & 2.8 & 0.64 & 0.53 \\
& HP & $10.5 \pm 1.95$ & & & \\
7 & SP & $11.5 \pm 2.17$ & & & \\
& CP & $8.4 \pm 1.58$ & 1.73 & 0.49 & \\
& HP & $8.6 \pm 2.06$ & & & \\
& SP & $9.2 \pm 1.93$ & & & \\
& CP & $7.2 \pm 1.61$ & 3.1 & 0.85 & \\
& HP & $7.9 \pm 2.33$ & & & \\
\hline
\end{tabular}

$\mathrm{CP}$, carbamide peroxide; $\mathrm{HP}$, hydrogen peroxide; $\mathrm{SP}$, sodium peroxide

Table 4 shows the shade variations in permanent and primary teeth with HP at 0,7 , and 14 days intervals. The shade for the both permanent and primary teeth shows no significant difference at the start of the study $(p=0.63)$. The permanent and primary teeth have showed no the significant difference even at the 7 days $(p=0.85)$ and 14 days $(p=0.83$ ) after the placement of medicaments.

Table 5 shows shade variations by the three experimental materials at 0,7 , and 14 days in permanent teeth. The three experimental materials have shown no significant variations in the shade variations at the baseline 0 days $(p=0.89)$. A statistically significant difference was observed between the three experimental at the 7 days $(p=0.00)$ and 14 days $(p=0.00)$ after the placement of medication in the permanent teeth.

Table 6 shows shade variations by the three experimental materials at 0,7 , and 14 days in primary teeth. The three experimental materials have shown no significant variations in the shade variations at the baseline 0 days $(p=0.53)$. No statistically significant difference was observed between the three experimental at the 7 days $(p=0.61)$ and 14 days $(p=0.44)$ after the placement of medication in the primary teeth.

\section{Discussion}

The intracoronal bleaching technique was introduced by Spasser, who employed a paste made up of SP and water, which was temporarily placed in the pulp chamber. This technique was modified by Nutting and Poe, who replaced water by superoxol. Stewart described the thermocatalytic method, in which a cotton pellet saturated with superoxol should be placed in the pulp chamber and heated with an instrument. ${ }^{13}$

According to Lim et al., it is well established that visual color determination is subjective and can be compared to the accuracy of spectrophotometer evaluation. ${ }^{8}$ Vachon et al. suggested that although the spectrophotometer readings might indicate a statistically significant difference, these differences could be clinically identical to the human eye. ${ }^{14}$ Thus, in the present study, the color changes in each specimen were evaluated using the Vita shade guide under standardized lighting conditions.

Placement of cervical seal after the endodontic treatment of the tooth is important in intracoronal bleaching technique so that the bleaching agent does not leach to the root, thus causing root resorption. ${ }^{15}$ 
The etiology of external cervical root resorption is unknown but it has been suggested that passage of bleaching agents to the periodontal tissues through the dentinal tubules would cause an inflammatory process around the teeth. ${ }^{15}$ Utilization of a cervical intermediate base following root filling is advocated for the prevention of external cervical root resorption in order to avoid penetration of the bleaching agent in apical and lateral directions. ${ }^{16}$ It was also recommended the placement of a protective base before intracoronal bleaching to prevent possible risks associated with $\mathrm{HP}^{17}$ In the present study, a glass ionomer cement was applied over the canal filling to prevent the risks of cervical resorptions in primary and permanent teeth.

Carrasco et al. reported that the $37 \%$ CP as an intracoronal bleaching increased dentinal permeability, which was not observed for $27 \% \mathrm{CP} .^{18}$ Thus, the use of $\mathrm{CP}$ at lower concentrations should be recommended to reduce the frequency of cervical root resorptions. ${ }^{19}$ The degradation of $10 \% \mathrm{CP}$ releases $6.4-7 \%$ of urea and $3-3.6 \%$ of $\mathrm{HP}$ where as the $35 \% \mathrm{CP}$ releases $23-25 \%$ of urea and $10-12 \%$ of HP. ${ }^{20}$ According to Braun et al., the high concentration of bleaching agent promotes faster tooth bleaching; however, considering the biological aspects, it is recommended to use the low-concentration agent to avoid damage tissue. ${ }^{21}$

Lim et al. concluded that $35 \%$ CP could be recommended as an effective alternative to HP. However, in this concentration, the biological effects of CP to dental and periodontal tissues should be more studied. ${ }^{8}$ Shaheen et al. have evaluated the efficiency of $10 \%$ $\mathrm{CP}$ in the primary teeth and observed that intracoronal bleaching using $10 \% \mathrm{CP}$ is an effective approach for whitening discolored extracted primary teeth. ${ }^{22}$ Thus, in the present study, $10 \%$ CP gel was used in comparison with the SP mixed with water and 10\% HP.

In the present study, 10\% CP have shown the lowest shade values after 7 days and 14 days in both the permanent and primary teeth groups. The present study results show that the $10 \% \mathrm{CP}$ is the effective bleaching agent in both permanent and primary teeth. The present study results are in accordance with the Tam, who observed that the $10 \%$ CP showed a clinically significant color changes in the permanent teeth within a week. ${ }^{23}$

The results of the present study are in accordance with Lim et al., who reported that though the effective HP released was less with $\mathrm{CP}$, it was equally effective to HP as a bleaching agent. ${ }^{8}$ The effectiveness of CP can also be attributed to its ability to improve the dentin permeability and to penetrate deeper, which further allows the reversal of chromatic alteration of the dental tissue by oxidation reactions. ${ }^{24}$ In the present study, CP has shown better lightening effect than by the SP, which was in accordance with Carrasco et al. who reported that the dentin permeability of $\mathrm{CP}$ is also higher than of the SP, which proves the better lightening effects of CP than of the SP. ${ }^{18}$ However, the present study results are contradictory to the reports of Perrine et al. who observed no statistical significant difference between the 10\% CP and SP in the tooth whitening. ${ }^{25}$

In the present study, the $\mathrm{CP}$ showed a significant difference between the lightening of permanent and primary teeth after 7 days and 14 days as well. Interestingly, the CP is more effective in lightening the permanent teeth than the primary teeth. The present study results are in accordance with the Ganesh et al. who observed that the HP is more effective than the CP in the primary teeth. ${ }^{26}$ Thus, the present study infers that though CP is more effective than HP and SP, it is more effective in permanent teeth than the primary teeth. The present study results also infer that both the HP and SP are equally effective in permanent and primary teeth with no statistical difference over 7 days and 14 days. The findings infer that the structural differences between the permanent and primary teeth do not affect the bleaching efficiency of HP or the SP in the permanent and primary teeth.

Though every effort was taken to imitate the exact clinical conditions in the present study, increasing the number of teeth per group might improve reliability of the results. The authors further recommend the in vivo or randomized controlled trials for more reliable results for treating the discolored anterior teeth.

\section{Conclusion}

The $\mathrm{CP}$ was considered as an effective bleaching agent in the treatment of discolored permanent teeth. The CP, HP, and SP are equally effective in treating the discolored primary teeth.

\section{References}

1. Marin PD. Intra-coronal bleaching: the past, present and future. Ann R Australas Coll Dent Surg 2006;18:36-39.

2. Azer SS, Hague AL, Johnston WM. Effect of bleaching on tooth discolouration from food colourant in vitro. J Dent 2011;39(Suppl 3):e52-e56. DOI: 10.1016/j.jdent.2011.09.006.

3. Schemel-Suárez M, López-López J, Chimenos-Küstner E. Dental pigmentation and hemochromatosis: a case report. Quintessence Int 2017;48(2):155-159. DOI: 10.3290/j.qi.a37385.

4. Kahler B, Rossi-Fedele G, Chugal N, et al. An evidence-based review of the efficacy of treatment approaches for immature permanent teeth with pulp necrosis. J Endod 2017;43(7):1052-1057. DOI: 10.1016/ j.joen.2017.03.003.

5. Bleaching of discoloured teeth. In: Chandra BS, Krishna VG, ed. Grossmans Endodontic Practice, 12th ed., ch. 14 New Delhi: Wolters Kluwer; 2010. p. 342.

6. Bizhang M, Domin J, Danesh G, et al. Effectiveness of a new nonhydrogen peroxide bleaching agent after single use - a double-blind placebo-controlled short-term study. J Appl Oral Sci 2017;25(5): 575-584. DOI: 10.1590/1678-7757-2016-0463.

7. Lee GP, Lee MY, Lum SO, et al. Extraradicular diffusion of hydrogen peroxide and $\mathrm{pH}$ changes associated with intracoronal bleaching of discoloured teeth using different bleaching agents. J Endod 2004;37(7):500-506. DOI: 10.1111/j.1365-2591.2004.00838.x.

8. Lim MY, Lum SO, Poh RS, et al. An in vitro comparison of the bleaching efficacy of 35\% carbamide peroxide with established intracoronal bleaching agents. Int Endod J 2004;37(7):483-488. DOI: 10.1111/j.13652591.2004.00829.x.

9. Gökay O, Ziraman F, Cali Asal A, et al. Radicular peroxide penetration from carbamide peroxide gels during intracoronal bleaching. Int Endod J 2008;41(7):556-560. DOI: 10.1111/j.1365-2591.2008.01384.x.

10. Sulieman M, MacDonald E, Rees JS, et al. Tooth bleaching by different concentrations of carbamide peroxide and hydrogen peroxide whitening strips: an in vitro study. J Esthet Restor Dent 2006;18(2): 93-100. DOI: 10.2310/6130.2006.00016_1.x.

11. Sonis AL. Longitudinal study of discolored primary teeth and effect on succedaneous teeth. J Pedod 1987;11(3):247-252.

12. Holan G, Fuks AB. The diagnostic value of coronal dark-gray discoloration in primary teeth following traumatic injuries. Pediatr Dent 1996;18(3):224-227.

13. Friedman S. Internal bleaching: long term outcomes and complications. J Am Dent Assoc 1997;128(Suppl):51S-55S. DOI: 10.14219/jada.archive.1997.0425.

14. Vachon C, VanekP, Friedman S. Internal bleaching with $10 \%$ carbamide peroxide in vitro. Pract Period Aesthet Dent 1998;10(9):1145-1148.

15. Oliviera LD, Carvalho CAT, Hilgert E, et al. Sealing evaluation of the cervical base in intracoronal bleaching. Dent Traumatol 2003;19(6):309-313. DOI: 10.1046/j.1600-9657.2003.00169.x.

16. Zarenejad N, Asgary S, Ramazani N, et al. Coronal microleakage of three different dental biomaterials as intra-orifice barrier during 
nonvital bleaching. Dent Res J (Isfahan) 2015;12(6):581-588. DOI: 10.4103/1735-3327.170582.

17. Sharma DS, Sharma S, Natu SM, et al. An in vitro evaluation of radicular penetration of hydrogen peroxide from bleaching agents during intra-coronal tooth bleaching with an insight of biologic response. J Clin Pediatr Dent 2011;35(3):289-294. DOI: 10.17796/jcpd.35.3.q83 $063560 \times 866037$.

18. Carrasco LD, Fröner IC, Corona SA, et al. Effect of internal bleaching agents on dentinal permeability of non-vital teeth: quantitative assessment. Dent Traumatol 2003;19(2):85-89. DOI: 10.1034/j.16009657.2003.00112.x.

19. Carrasco LD, Zanello Guerisoli DM, Pécora JD, et al. Evaluation of dentin permeability after light activated internal dental bleaching. Dent Traumatol 2007;23(1):30-34. DOI: 10.1111/j.1600-9657.2006.00469.x.

20. Chng HK. Update on materials used in intracoronal bleaching. Ann R Australas Coll Dent Surg 2002;16:147-150.

21. Braun A, Jepsen S, Krause F. Spectrophotometric and visual evaluation of vital tooth bleaching employing different carbamide peroxide concentrations. Dent Mater 2007;23(2):165-169. DOI: 10.1016/j. dental.2006.01.017.

22. Shaheen MA, Elkateb MA, Bakry NS, et al. Efficacy of 10 percent carbamide peroxide as an intracoronal bleaching agent in nonvital discolored primary teeth: an in vitro study. J Dent Child (Chic) 2017;84(1):22-29.

23. Rotstein I, Dankner E, Goldman A, et al. Histochemical analysis of dental hard tissues following bleaching. J Endod 1996;22(1):23-25. DOI: 10.1016/S0099-2399(96)80231-7.

24. Tam L. Clinical trial of three $10 \%$ carbamide peroxide bleaching products. J Can Dent Assoc 1999;65(4):201-205.

25. Perrine GA, ReichI RB, Baisden MK, et al. Comparison of $10 \%$ carbamide peroxide and sodium perborate for intracoronal bleaching. Gen Dent 2000;48(3):264-270.

26. Ganesh R, Aruna S, Joyson M, et al. Comparison of the bleaching efficacy of three different agents used for intracoronal bleaching of discolored primary teeth: an in vitro study. J Indian Soc Pedod Prev Dent 2013;31(1):17-21. DOI: 10.4103/0970-4388.112394. 